\title{
Paramètres morphobiométriques et biochimiques des chevaux de traction au Sénégal
}

\author{
A. BATHILY, A. SOW*, M. KALANDI et G.J. SAWADOGO \\ Ecole Inter-Etats des Sciences et Médecine Vétérinaires (EISMV), Laboratoire d'Endocrinologie et de Radio- \\ Immunologie, BP. 5077, Dakar Fann, Sénégal. Tel. (+221) 3386510 08, Fax. (+221) 338254283 , \\ *Auteur correspondant, E-mail: wosamada@yahoo.fr ; a.sow@eismv.org
}

\section{RESUME}

Le cheval de traction joue un important rôle dans l'agriculture et le transport interurbain. Cependant, il existe peu de données sur les paramètres morphobiométriques et biochimiques des chevaux locaux. Ces données sont pourtant nécessaires pour une prise en charge clinique. Cette étude visait la détermination de ces paramètres. Pour ce faire, 97 chevaux mâles ont été sélectionnés dans les Régions de Kaolack et Saint-Louis. Les chevaux étaient répartis en 3 groupes d'âge : G1 [2-3ans], G2 [4-9ans] et G3 [10-27ans] et en deux groupes selon la note d'état corporel (NEC). Les paramètres morphobiométiques ont été mesurés à l'aide d'une toise et d'un mètre-ruban. Tandis que les paramètres biochimiques ont été dosés à l'aide de kits commerciaux $\left(\right.$ Biosystem $\left.^{\circledR}\right)$. Des variations ont été notées dans les valeurs des paramètres biochimiques et morphobiométriques. Ainsi, les valeurs du phosphore, du calcium, du cholestérol, de l'urée, de l'ASAT et de la créatinine ont varié significativement selon la région $(\mathrm{p}<0,05)$. De même, la hauteur au garrot et de la Longueur du tronc ont varié en fonction de la localité ( $\mathrm{p}<0,001)$. Les valeurs de l'urée, de la créatinine, du calcium et du phosphore ont varié significativement avec l'âge $(\mathrm{p}<0,05)$. L'établissement des paramètres biochimiques permettra une meilleure prise en charge clinique des chevaux de traction au Sénégal.

(C) 2014 International Formulae Group. All rights reserved.

Mots clés : Cheval de traction, paramètres morphobiométriques, paramètres biochimiques, Sénégal.

\section{INTRODUCTION}

L'utilisation des animaux en Afrique a une longue histoire. Les chevaux de traction jouent un rôle important dans la production agricole et le transport en Afrique subsaharienne (Pearson et Lhoste, 2003). L'utilisation de transport par traction animale a été d'abord introduite dans les ports côtiers et continentaux d'Afrique du XVII ${ }^{\text {ème }}$ au $\mathrm{XIX}^{\mathrm{ème}}$ siècle. Lorsque les conditions sociales, économiques et écologiques se sont avérées favorables, la technologie s'est graduellement répandue à l'intérieur du continent africain, à travers les activités des commerçants, des colonisateurs, des missionnaires et des autorités administratives. Les équidés (chevaux, ânes et mules) et les bœufs ont été utilisés pour le transport des personnes et des biens (Starkey, 2004).

Le cheptel équin du Sénégal est estimé à 534124 têtes (DIREL, 2012). Le cheval joue un rôle socio-économique très important. Ainsi, le cheval de trait agricole et la traction hippomobile jouent toujours un rôle fondamental dans les relations et les échanges ruraux, la commercialisation des produits agricoles, le transport de matériaux de construction et la collecte des ordures domestiques (Akpo, 2004).

Malgré cette grande importance, peu de travaux ont été réalisés sur les paramètres morphobiométriques et biochimiques des races locales de chevaux au Sénégal. Les analyses de biochimie sanguine ont pour 
objectif d'aider le praticien à poser le diagnostic les plus fiables des maladies des équins.

C'est dans ce contexte que s'est réalisée cette étude avec pour principal objectif d'évaluer les paramètres morphobiométriques et biochimiques des chevaux et déterminer les facteurs de variations de ces paramétres.

\section{MATERIEL ET METHODES \\ Sites de l'étude}

L'étude a été menée en avril et juillet dans le bassin arachidier (région de Kaolack) et dans la zone sylvopastorale (région de Saint Louis), deux régions agricoles par excellence du Sénégal (Figure 1). Les températures moyennes pendant la période de l'étude variaient entre $35-40{ }^{\circ} \mathrm{C}$.

Dans les régions de Kaolack et SaintLouis, les pluviométries annuelles varient en moyenne entre $850 \mathrm{~mm}$ et $346 \mathrm{~mm}$ respectivement. Pour les deux régions, les activités agricoles occupent $75 \%$ de la population et s'adonnent aux cultures vivrières (riz, mil et maïs), les cultures de rentes (arachide et sésame) et des cultures maraîchères.

\section{Sélection des animaux}

Quatre-vingt-dix-sept chevaux mâles de race locale de traction âgés de 2 à 27 ans, dont 47 dans la région de Kaolack et 50 dans la région de Saint-Louis ont été sélectionnés. Pour une bonne interprétation de l'influence de l'âge sur les paramètres biochimiques, les animaux de l'étude étaient répartis en 3 groupes d'âge : groupe 1([2 - 3 ans]); groupe 2 ([4 - 9 ans]); groupe 3([10 -27 ans]). Les moyennes d'âge étaient 2,58 $\pm 0,51$ ans ; $6,20 \pm 1,43$ ans; $13,45 \pm 3,8$ ans respectivement pour les groupes 1,2 et 3 . Pour tous les chevaux, la note d'état corporel a été évaluée selon une échelle de 0 à 5 (Evans, 2010) et deux groupes ont été identifiés: les chevaux à $\mathrm{NEC}<3$ et à $\mathrm{NEC} \geq 3$. Les donnnées portant sur l'âge, la race, la NEC ont été collectées et enregistrées à l'aide d'une fiche d'enquête. Tous les chevaux de cette étude étaient des sujets mâles entiers de race locale, âgés de plus de 2 ans et apparemment en bonne santé (après un examen clinique). Les métis, les femelles, les sujets très jeunes et les chevaux de sport n'ont pas été choisis. De même, les animaux présentant une affection susceptible de modifier les résultats des prélèvements sanguins ont été exclus de l'étude.

\section{Détermination des paramètres morphobiométiques}

Pour chaque cheval des mesures barymétriques telles que la hauteur au garrot (HG), la longueur du tronc (LT) et le périmètre thoracique (PT) ont été relevées pour la caractérisation morphobiométrique. La HG a été mesurée à l'aide d'une toise à niveau au point le plus haut du garrot, cheval étant sur une surface plane et avec une hauteur de tête normale. La LT a été évaluée avec un ruban-mètre de la pointe de l'épaule (processus de l'olécrane) à la pointe de la fesse (pointe de l'ischium). Le PT a été également déterminé à l'aide d'un rubanmètre au niveau du passage de sangle après l'expiration.

\section{Détermination du profil biochimique des chevaux sélectionnés}

Des prélèvements sanguins ont été réalisés sur tous les animaux de l'étude par ponction de la veine jugulaire. Le sang a été collecté dans des tubes secs stériles sous vide (Vacutainer $^{\circledR}$ ) portant le nom ou numéro de l'animal. Les échantillons sanguins ont été centrifugés (3500 tours/min, 15 minutes) et les sérums recueillis ont été conservés à-20 ${ }^{\circ} \mathrm{C}$ jusqu'au jour de l'analyse. Les analyses biochimiques ont concerné les métabolites (protéines totales, l'albumine, créatinine et urée), les enzymes l'aspartate aminotransférase (ASAT) et l'alanine aminotransférase (ALAT), les ions (magnésium, phosphore et le calcium) et le cholestérol.Les analyses biochimiques ont été réalisées en utilisant des kits commerciaux (BIOSYSTEMS $^{\circledR}$, S.A., Barcelona, Spain). Les dosages ont été effectués selon le principe décrit par les laboratoires Biosystems ${ }^{\circledR}$. Les réactions colorimétriques ont été mesurées à l'aide d'un spectrophotomètre (BIOSYSTEMS BTS $^{\circledR}$ ). De même, le profil électrophorétique a été déterminé à l'aide de kits commerciaux SEBIA ${ }^{\circledR}$. 


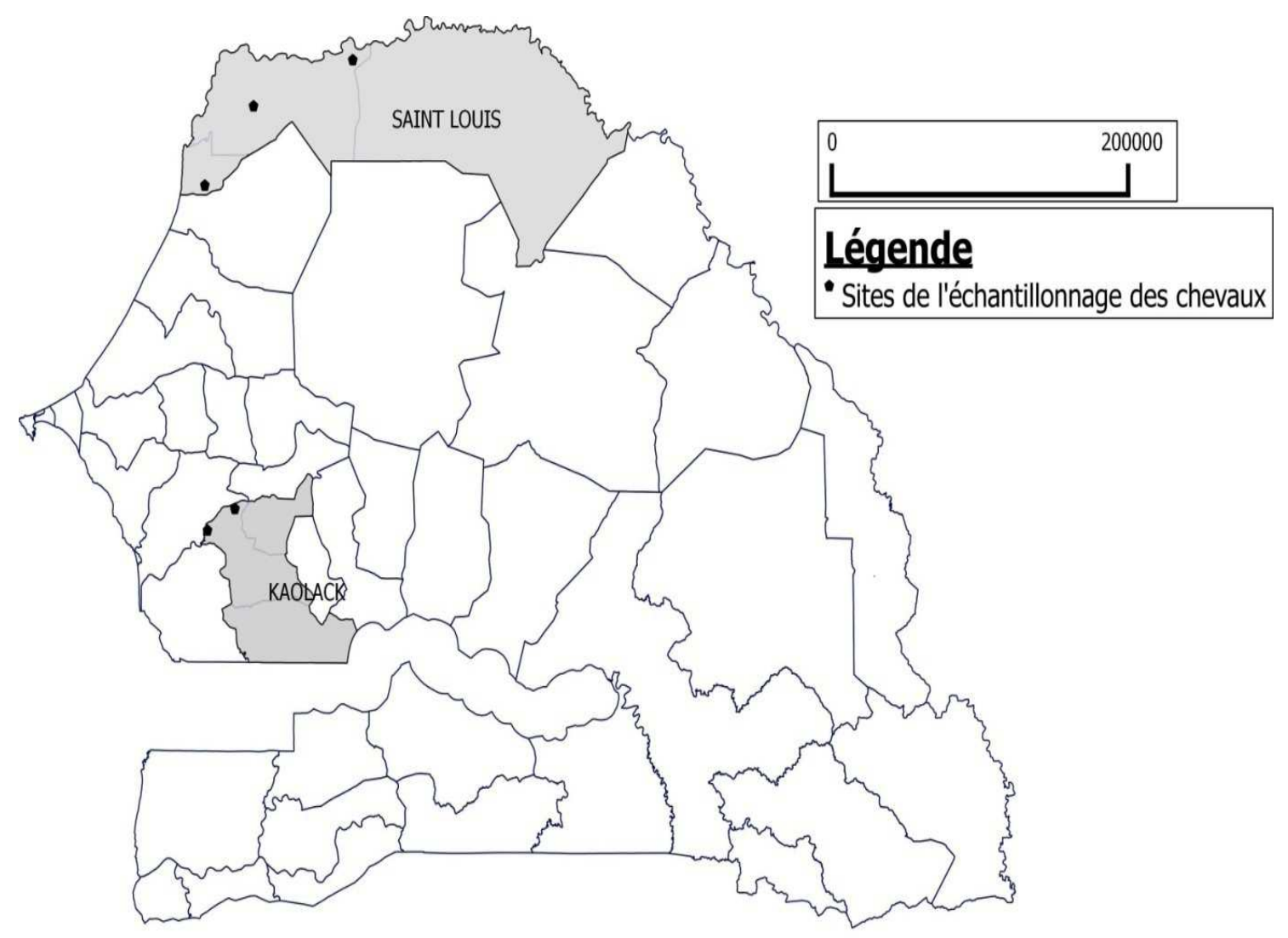

Figure 1 : Localisation des régions de Kaolack et de Saint-Louis, site de l'étude.

\section{Analyses statistiques}

Les données ont été analysées avec le logiciel STATA S.E. 9.2 ${ }^{\circledR}$. Pour chaque paramètre, la moyenne et l'écart type ont été calculés. Le t-test de Student et l'ANOVA ont été utilisés pour comparer les écarts entre les valeurs moyennes des paramètres obtenues (groupe d'âge, localité et la NEC). Les différences ont été considérées statistiquement significatifs à $\mathrm{p}<0,05$.

\section{RESULTATS}

\section{Caractérisation de la population d'étude}

La NEC moyenne a été de 2,9 $\pm 0,8$ et 2,3 $\pm 0,8$ pour les chevaux de Kaolack et de Saint-Louis respectivement. Les moyennes d'âge étaient de 7,3 $\pm 5,4$ ans et $8 \pm 3,8$ ans pour la région de Kaolack et de SaintLouisrespectivement. Toutefois une variation de la NEC selon l'age a été observée (Tableau 1); mais les variations n'ont pas été significatives entre les différents groupes d'âge pour une même localité ( $p>0,05)$.

\section{Paramètres morphobiométriques}

Le Tableau 2 présente les paramètres morphobiométriques moyennes obtenus dans la population étudiée. Ces paramètres varient selon l'âge, la localité et la NEC. L'âge a eu un effet significatif sur les valeurs moyennes de la longueur du tronc $(\mathrm{p}=0,022)$ et $\mathrm{du}$ pourtour thoracique $(\mathrm{p}=0,002)$. Toutefois, il n'y a pas eu de différence significative entre les valeurs moyennes de ces 2 paramètres entre les groupe 2 et 3 ( $p>0.05)$. Un effet significatif de la localité sur les valeurs moyennes de la hauteur au garrot $(\mathrm{p}=0,000)$ et de la longueur du tronc $(\mathrm{p}=0,000)$ a été noté. La valeur moyenne de la hauteur au garrot des chevaux de la région de Kaolack était supérieure à celle de la région de Saint-Louis $(\mathrm{p}<0,05)$. De même, la valeur moyenne de la longueur du tronc des animaux de la région de 
Kaolack était inférieure à celle de la région de Saint-Louis $(\mathrm{p}<0,05)$. La NEC a eu un effet significatif sur les valeurs moyennes de la hauteur au garrot $(\mathrm{p}=0.000)$, du pourtour thoracique $(\mathrm{p}=0,000)$ et la longueur du tronc $(p=0,004)$. Les moyennes de la hauteur au garrot et du pourtour thoracique des chevaux de $\mathrm{NEC}<3$ étaient inférieures à celles des animaux de $\mathrm{NEC} \geq 3$. La valeur moyenne de la longueur du tronc des animaux de $\mathrm{NEC}<3$ était supérieure à celle des chevaux de $\mathrm{NEC} \geq 3$.

\section{Paramètres biochimiques}

Les valeurs moyennes des paramètres biochimiques pour la population totale sont résumées dans le Tableau 3. Les valeurs des paramètres biochimiques trouvés sont dans le même ordre de grandeur que celles qui ont été établies dans les études antérieures sur le cheval en général.

Variations des paramètres biochimiques selon l'âge

L'âge a eu un effet significatif sur les valeurs de l'urée $(\mathrm{p}=0,021)$, de la créatinine $(p=0,024), d u$ calcium $(p=0,007)$ et $d u$ phosphore $(\mathrm{p}=0,000)$ (Tableau4). La moyenne de l'urémie des chevaux du groupe 1 était inférieure à celles des groupes 2 et 3 . Toutefois, il n'y a pas eu de différence significative entre les valeurs moyennes de ce paramètre entre les groupes 2 et 3 ( $p>0,05)$. De même, la valeur moyenne de la créatinine des animaux du groupe 1 était inférieure à celles des groupes 2 et 3 . Et une différence significative des moyennes de la créatinine entre les groupes 2 et 3 a été notée. Les valeurs moyennes du calcium et du phosphore des chevaux du groupe 1 étaient supérieures à celles des groupes 2 et 3. Mais il n'y a pas eu de différence significative de la calcémie et de la phosphorémie entre les groupes 2 et 3 ( $\mathrm{p}$ > 0,05).

\section{Variations des paramètres biochimiques selon la localité}

Les moyennes de l'ASAT, l'urée, de la créatinine, du phosphore et du cholestérol des chevaux de la région de Saint-Louis étaient supérieures à celles des animaux de la région de Kaolack $(\mathrm{p}=0,05)$. Par contre, les valeurs moyennes des protéines totales et du calcium obtenues chez les chevaux de la région de Kaolack étaient supérieures à celles des animaux de la région de Saint-Louis ( $\mathrm{p}=0,047$; Tableau 5).

Variations des paramètres biochimiques selon la NEC

Il n y a pas eu d'effet significatif de la NEC sur les paramètres biochimiques excepté la créatinine ( $p=0,047$; Tableau 6). La valeur moyenne de la créatinine chez les chevaux de $\mathrm{NEC}<3$ était supérieure à celle des animaux de $\mathrm{NEC} \geq 3$.

Tableau 1 : Répartition des chevaux en fonction de la NEC, de l'âge et de la localité.

\begin{tabular}{lcccccccc}
\hline Facteurs de & \multicolumn{4}{c}{ Kaolack } & \multicolumn{3}{c}{ Saint-Louis } \\
\cline { 2 - 8 } variations & G1(14) & G2(21) & G3(12) & Total(47) & G1(5) & G2(28) & G3(17) & Total(50) \\
\hline NEC & $2,6 \pm 0,5$ & $3 \pm 0,9$ & $2,8 \pm 0,9$ & $2,9 \pm 0,8^{\mathrm{a}}$ & $2,2 \pm 0,4$ & $2,3 \pm 0,8$ & $2,3 \pm 0,9$ & $2,3 \pm 0,8^{\mathrm{b}}$ \\
Age (ans) & $2,6 \pm 0,5$ & $6 \pm 1,5$ & $15 \pm 4,8$ & $7,3 \pm 5,4^{\mathrm{a}}$ & $2,6 \pm 0,5$ & $6,3 \pm 1,3$ & $12,4 \pm 2,5$ & $8 \pm 3,8^{\mathrm{a}}$ \\
\hline
\end{tabular}

Lettres $(a, b)$ différents sur une même ligne=différence significative $(\mathrm{p}<0,05)$. 
A. Bathily et al. /Int. J. Biol. Chem. Sci. 8(6): 2731-2739, 2014

Tableau 2 : Moyennes des paramètres morphobiométriques de la population totale en fonction du groupe d'âge, de la localité et de la NEC.

\begin{tabular}{|c|c|c|c|c|c|c|c|c|}
\hline \multirow[b]{2}{*}{ Paramètres (cm) } & \multirow[b]{2}{*}{$\begin{array}{c}\text { Total } \\
(\mathbf{n}=97)\end{array}$} & \multicolumn{3}{|c|}{ Age } & \multicolumn{2}{|c|}{ Localité } & \multicolumn{2}{|c|}{ NEC } \\
\hline & & $\begin{array}{c}\text { Groupe1 } \\
(n=19)\end{array}$ & $\begin{array}{c}\text { Groupe } 2 \\
(n=49)\end{array}$ & $\begin{array}{c}\text { Groupe3 } \\
(\mathrm{n}=29)\end{array}$ & $\begin{array}{c}\text { Kaolack } \\
(n=47)\end{array}$ & $\begin{array}{c}\text { Saint-Louis } \\
(\mathbf{n}=50)\end{array}$ & $\begin{array}{c}\mathrm{NEC}<3 \\
(\mathrm{n}=46)\end{array}$ & $\mathrm{NEC} \geq 3(\mathrm{n}=51)$ \\
\hline Hauteur au garrot & $136,6 \pm 5,6$ & $134,3 \pm 5,7^{\mathrm{a}}$ & $137,7 \pm 5,8^{\mathrm{a}}$ & $136,3 \pm 4,6^{\mathrm{a}}$ & $138,8 \pm 5,3^{\mathrm{a}}$ & $134,6 \pm 5^{b}$ & $134,6 \pm 5,3^{\mathrm{a}}$ & $138,5 \pm 5,2^{b}$ \\
\hline Longueur tronc & $122 \pm 15,5$ & $113,4 \pm 10,9^{\mathrm{a}}$ & $124,6 \pm 15,7^{\mathrm{b}}$ & $123,2 \pm 16,2^{\mathrm{c}}$ & $108,3 \pm 8,6^{\mathrm{a}}$ & $134,9 \pm 7,4^{\mathrm{b}}$ & $126,3 \pm 13,1^{\mathrm{a}}$ & $118,1 \pm 16,6^{\mathrm{b}}$ \\
\hline Périmètre thoracique & $146,9 \pm 8,6$ & $141,4 \pm 8,1^{\mathrm{a}}$ & $149,3 \pm 8,6^{\mathrm{b}}$ & $146,2 \pm 7,1^{\mathrm{c}}$ & $148,3 \pm 8,7^{\mathrm{a}}$ & $145,7 \pm 8,3^{\mathrm{a}}$ & $141,7 \pm 6,2^{\mathrm{a}}$ & $151,6 \pm 7,6^{\mathrm{b}}$ \\
\hline
\end{tabular}

Tableau 3 : Moyennes des paramètres biochimiques pour l'ensemble des chevaux échantillonnés.

\begin{tabular}{|c|c|c|}
\hline Paramètres & Population totale $(n=97)$ & Valeurs de reference \\
\hline ALAT (UI/l) & $8,75 \pm 9.9$ & $25.24 \pm 5.8^{\mathrm{a}}$ \\
\hline ASAT (UI/l) & $135 \pm 57.4$ & $229.67 \pm 46.8^{\mathrm{a}}$ \\
\hline Urée (mmol/l) & $5.07 \pm 1.8$ & $2,2-7,3^{\mathrm{b}}$ \\
\hline Créatinine $(\mu \mathrm{mol} / \mathrm{l})$ & $128,84 \pm 26.4$ & $78,3-157,7^{\mathrm{c}}$ \\
\hline Protéines totales $(\mathrm{g} / \mathrm{l})$ & $61,44 \pm 6$ & $69,90 \pm 6,7^{\mathrm{d}}$ \\
\hline Albumine $(\mathrm{g} / \mathrm{l})$ & $28,15 \pm 4.61$ & $32.45 \pm 8.4^{\mathrm{d}}$ \\
\hline Globulines $(\mathrm{g} / \mathrm{l})$ & $39,38 \pm 5,8$ & $26.2-40.4^{\mathrm{e}}$ \\
\hline Globulines alpha1 (g/l) & $2,78 \pm 0,4$ & $0.6-7.0^{\mathrm{e}}$ \\
\hline Globulines alpha2 (g/l) & $8,19 \pm 0,9$ & $3.1-13.1^{\mathrm{e}}$ \\
\hline Globulines beta $(\mathrm{g} / \mathrm{l})$ & $11,16 \pm 2.9$ & $9-18^{\mathrm{e}}$ \\
\hline Globulines gamma $(\mathrm{g} / \mathrm{l})$ & $12,70 \pm 3.3$ & $5.5-19.0^{\mathrm{e}}$ \\
\hline Albumine/globulines & $0,92 \pm 0,5$ & $0.57-1.42^{\mathrm{c}}$ \\
\hline Magnésium (mmol/l) & $1.02 \pm 0.2$ & $0.90-1.15^{\mathrm{e}}$ \\
\hline Calcium $(\mathrm{mmol} / \mathrm{l})$ & $2,92 \pm 0,31$ & $2,67-3,09$ \\
\hline Phosphore (mmol/l) & $1.47 \pm 0.6$ & $0.47-1.96^{\mathrm{c}}$ \\
\hline Cholestérol (mmol/l) & $2,20 \pm 0,3$ & $1.94-3.89^{\mathrm{e}}$ \\
\hline
\end{tabular}


Tableau 4 : Moyennes des paramètres biochimiques en fonction des groupes d'âge.

\begin{tabular}{|c|c|c|c|c|c|}
\hline Paramètres & $\begin{array}{c}\text { Groupe } 1 \\
n=19\end{array}$ & $\begin{array}{c}\text { Groupe } 2 \\
n=49\end{array}$ & $\begin{array}{c}\text { Groupe } 3 \\
\mathbf{n}=29\end{array}$ & $\mathbf{F}$ & p-value \\
\hline ALAT (UI/l) & $5,48 \pm 4,8$ & $8,84 \pm 10,3$ & $10,73 \pm 11,2$ & 1,63 & 0,200 \\
\hline ASAT (UI/l) & $122,13 \pm 40,1$ & $140,36 \pm 65,3$ & $134,37 \pm 52,4$ & 0,69 & 0,505 \\
\hline Urée $(\mathrm{mmol} / \mathrm{l})$ & $4,02 \pm 1,5$ & $5,34 \pm 1,9$ & $5,30 \pm 1,7$ & 4,03 & $0,021^{*}$ \\
\hline Créatinine $(\mu \mathrm{mol} / \mathrm{l})$ & $121,08 \pm 18,7$ & $125,45 \pm 23,5$ & $139,66 \pm 32,3$ & 3,86 & $0,024^{*}$ \\
\hline Protéines totales $(\mathrm{g} / \mathrm{l})$ & $62,05 \pm 5,4$ & $60,17 \pm 5,2$ & $63,18 \pm 7,2$ & 2,47 & 0,089 \\
\hline Albumine $(\mathrm{g} / \mathrm{l})$ & $27,05 \pm 2,4$ & $28,43 \pm 4,8$ & $28,40 \pm 5,3$ & 0,66 & 0,516 \\
\hline Magnésium (mmol/l) & $1,01 \pm 0,25$ & $0,99 \pm 0,28$ & $1,08 \pm 0,35$ & 0,67 & 0,512 \\
\hline Calcium (mmol/l) & $3,12 \pm 0,31$ & $2,88 \pm 0,29$ & $2,86 \pm 0,29$ & 5,17 & $0,007^{*}$ \\
\hline Phosphore (mmol/l) & $1,95 \pm 0,7$ & $1,36 \pm 0,5$ & $1,34 \pm 0,5$ & 6,74 & $0,000^{* *}$ \\
\hline Cholestérol (mmol/l) & $2,34 \pm 0,4$ & $2,13 \pm 0,3$ & $2,24 \pm 0,3$ & 2,94 & 0,057 \\
\hline
\end{tabular}

$* \mathrm{p}<0,05:$ différence significative, ${ }^{* *} \mathrm{p}<0,001:$ différence très significative.

Tableau 5 : Moyennes des paramètres biochimiques en fonction de la localité.

\begin{tabular}{lcccc}
\hline Paramètres & Kaolack $(\mathbf{n}=\mathbf{4 7})$ & Saint-Louis $(\mathbf{n}=\mathbf{5 0})$ & $\mathbf{T}$ & $\boldsymbol{p}$-value \\
\hline ALAT $(\mathrm{UI} / \mathrm{l})$ & $7,53 \pm 8,8$ & $9,89 \pm 10,7$ & 1,176 & 0,121 \\
ASAT $(\mathrm{UI} / \mathrm{l})$ & $110,04 \pm 53,1$ & $158,40 \pm 51,4$ & 4,557 & $0,000^{* *}$ \\
Urée $(\mathrm{mmol} / \mathrm{l})$ & $4,57 \pm 1,5$ & $5,54 \pm 1,9$ & 2,661 & $0,004^{*}$ \\
Créatinine $(\mu \mathrm{mol} / \mathrm{l})$ & $115,59 \pm 17,7$ & $141,3 \pm 27,3$ & 5,445 & $0,000^{* *}$ \\
Protéines totales $(\mathrm{g} / \mathrm{l})$ & $62,49 \pm 5,7$ & $60,45 \pm 6,1$ & 1,690 & $0,047^{*}$ \\
Albumine $(\mathrm{g} / \mathrm{l})$ & $27,94 \pm 3,7$ & $28,35 \pm 5,3$ & 0,427 & 0,334 \\
Magnésium $(\mathrm{mmol} / \mathrm{l})$ & $0,97 \pm 0,2$ & $1,07 \pm 0,3$ & 1,555 & 0,061 \\
Calcium $(\mathrm{mmol} / \mathrm{l})$ & $3,01 \pm 0,3$ & $2,84 \pm 0,2$ & 2,649 & $0,004^{*}$ \\
Phosphore $(\mathrm{mmol} / \mathrm{l})$ & $1,27 \pm 0,6$ & $1,65 \pm 0,6$ & 2,933 & $0,002^{*}$ \\
Cholestérol $(\mathrm{mmol} / \mathrm{l})$ & $2,09 \pm 0,2$ & $2,31 \pm 0,3$ & 3,226 & $0,000^{* *}$ \\
\hline *p<0,05: différence significative, $* * \mathrm{p}<0,001 \cdot$ différence très significative. & &
\end{tabular}

${ }^{*} \mathrm{p}<0,05$ : différence significative, ${ }^{* *} \mathrm{p}<0,001:$ différence très significative.

Tableau 6 : Moyennes des paramètres biochimiques en fonction de la NEC.

\begin{tabular}{lcccc}
\hline Paramètres & NEC $<\mathbf{3}(\mathbf{n}=\mathbf{4 6})$ & $\mathbf{N E C} \geq \mathbf{3}(\mathbf{n = 5 1})$ & $\mathbf{t}$ & p-value \\
\hline ALAT $(\mathrm{UI} / \mathrm{l})$ & $7,87 \pm 9,3$ & $9,54 \pm 10,4$ & 0,825 & 0,205 \\
ASAT $(\mathrm{UI} / \mathrm{l})$ & $132,17 \pm 50,1$ & $137,56 \pm 63,7$ & 0,459 & 0,323 \\
Urée $(\mathrm{mmol} / \mathrm{l})$ & $5,29 \pm 2,1$ & $4,87 \pm 1,5$ & 1,131 & 0,130 \\
Créatinine $(\mu \mathrm{mol} / \mathrm{l})$ & $133,57 \pm 30,6$ & $124,58 \pm 21,4$ & 1,685 & $0,047 *$ \\
Protéines totales $(\mathrm{g} / \mathrm{l})$ & $60,97 \pm 6,5$ & $61,86 \pm 5,5$ & 0,726 & 0,234 \\
Albumine $(\mathrm{g} / \mathrm{l})$ & $27,75 \pm 4,9$ & $28,52 \pm 4,2$ & 0,822 & 0,206 \\
Magnésium $(\mathrm{mmol} / \mathrm{l})$ & $1,06 \pm 0,3$ & $0,98 \pm 0,2$ & 1,356 & 0,089 \\
Calcium $(\mathrm{mmol} / \mathrm{l})$ & $2,93 \pm 0,3$ & $2,92 \pm 0,2$ & 0,051 & 0,479 \\
Phosphore $(\mathrm{mmol} / \mathrm{l})$ & $1,49 \pm 0,6$ & $1,44 \pm 0,6$ & 0,376 & 0,353 \\
Cholestérol $(\mathrm{mmol} / \mathrm{l})$ & $2,25 \pm 0,4$ & $2,15 \pm 0,3$ & 1,389 & 0,084 \\
\hline \multicolumn{2}{c}{$* 0,05:$ différence significative, $* * \mathrm{p}<0,001:$ différence très significative. } & &
\end{tabular}




\section{DISCUSSION}

Les chevaux de l'étude étaient tous de race locale, utilisés pour la traction. La population d'étude était composée seulement de chevaux mâles. Ceci se justifierait par le fait que les mâles sont constamment utilisés pour le travail agricole ou le transport. Les juments sont les moins sollicitées à cause de leur non utilisation pour le transport.

Les moyennes des paramètres morphobiométriques obtenues pour la population totale sont du même ordre de grandeur que celles trouvées dans des études antérieures sur les races locales de chevaux du Sénégal (Larrat, 1947). Cependant, ces valeurs sont inférieures à celles des chevaux barbe et dérivés du Maghreb (Guedaoura et al., 2011 ; Rahal et al., 2009).

La variation de certains paramètres morphobiométriques selon l'âge, serait due au fait que les jeunes chevaux ( $<4$ ans), toujours en phase de croissance, avaient un périmètre thoracique et une longueur du tronc significativement inférieurs aux valeurs des chevaux plus âgés. De même, le périmètre thoracique a varié selon l'état d'embonpoint des chevaux. En effet, les valeurs du pourtour thoracique des chevaux de NEC $\geq 3$ étaient supérieures à celles des animaux de NEC $<3$; ceci pourrait s'expliquer par le fait que les premiers avaient plus de réserves corporelles que les derniers.

La valeur moyenne de la hauteur au garrot des chevaux de la région de Kaolack était plus élevée que celle des animaux de la région de Saint-Louis. Cependant, la valeur moyenne de la longueur du tronc des animaux de la région de Kaolack était plus basse que celle des chevaux de la région de Saint-Louis. Les différences de la hauteur au garrot et de la longueur du tronc observées entre les chevaux des deux localités laissent présager une diversité phénotypique qui pourrait être confirmée par des méthodes de génétiques moléculaires. Il y a également la possibilité que les différences écoclimatiques pourraient avoir un impact sur l'interaction environnementale du génotype. Ces différences pourraient aussi être dues aux races élevées dans ces régions ou encore à la région de vie ou au biotope.
De manière générale, toutes les valeurs des paramètres biochimiques obtenues chez les chevaux de traction étaient dans le même ordre de grandeur des valeurs rapportées par des études antérieures (Bathily et al., 2013 ; Gul et al., 2007 ; Kaneko et al., 2008 ; Niedźwiedź et al., 2013 ; Tomenendalova et al., 2014).

La valeur plus basse de l'urée chez les jeunes chevaux ( $<4$ ans) pourrait s'expliquer par leur anabolisme protéique important lié à une croissance rapide (Cornus, 2010). En effet chez les jeunes animaux en croissance rapide l'anabolisme protéique est prédominant par rapport au catabolisme (Frandson et al., 2009). Le catabolisme protéique est donc faible durant cette période et logiquement la production d'urée l'est autant.

La valeur plus basse de la créatinémie chez les jeunes chevaux pourrait s'expliquer, par une masse musculaire plus réduite que celle des chevaux adultes. L'effet significatif de l'âge sur la valeur de l'urée et la créatinine a été rapporté par d'autres auteurs (Cornus, 2010; Ihedioha et Agina, 2013).

De même, le phosphore et le calcium ont varié en fonction des groupes d'âge. Les valeurs moyennes du phosphore et du calcium étaient plus élevées chez les jeunes chevaux. Cependant, des études antérieures ont démontré que la concentration de phosphore dans le sérum diminuait significativement avec l'âge chez les chevaux, les ânes et autres animaux domestiques (Gurgoze et Icen, 2010 ; Pritchard et al., 2009 ; Rumosa et al., 2012). Ces études ont suggéré que la diminution liée à l'âge reflète probablement une diminution du métabolisme des os chez les animaux âgés. En effet, la rétention et la digestibilité apparente du phosphore sont plus élevées chez les jeunes chevaux et diminuent avec l'âge (Ögren, 2013).

Les valeurs de l'ASAT, de l'urée, de la créatinine, du phosphore et du cholestérol des chevaux de la région de Saint-Louis étaient significativement supérieures à celles animaux de la région de Kaolack. Bien que les différences aient été significatives, toutes les valeurs de ces paramètres étaient dans les normes physiologiques. Les valeurs élevées de l'ASAT chez les chevaux de Saint-Louis pourraient être dues à une activité musculaire 
plus intense chez ces animaux que ceux de Kaolack. En effet, les enquêtes se sont déroulées en avril et en juillet respectivement à Kaolack et à Saint-Louis. Le mois d'avril est une période de repos pour les chevaux, majoritairement utilisés comme auxiliaires agricoles. Cependant, le mois de juillet est le début de la saison des pluies, et les chevaux sont beaucoup sollicités pour la préparation des champs. Hors il a été montré que si l'activité musculaire est importante, le niveau de l'ASAT dans le plasma augmente (Trigo et al., 2010).

La différence de la créatinémie serait due à la différence d'activité musculaire. La créatinémie plus élevée chez les animaux de Saint-Louis corrobore les variations de l'ASAT observées chez les chevaux de SaintLouis. Les chevaux de la région de SaintLouis avaient une activité musculaire plus importante, donc leur concentration en créatinine augmente. Or la masse musculaire et le niveau d'activité peuvent influer la production et donc la concentration de la créatinine dans le sang. Par ailleur, certains auteurs ont rapporté que la créatinémie était plus élevée chez les chevaux entraînés que chez les chevaux non entraînés (Petruse et Falca, 2008).

Les variations des autres paramètres à savoir l'urée, le cholestérol le calcium et le phosphore pourraient être dues à des facteurs nutritionnels. En effet, des facteurs nutritionnels peuvent influencer les concentrations plasmatiques de ces paramètres comme notés dans plusieurs études antérieures chez différentes espèces d'animaux (Carlson, 2002 ; Gálik et al., 2012 ; Simenew et al., 2011 ; Vervuert et al., 2006).

\section{Conclusion}

$\begin{array}{ccc}\text { Cette étude a permis de mieux } \\ \text { connaître } & \text { les } & \text { caractéristiques }\end{array}$ morphobiométriques des chevaux races locales et d'établir les valeurs usuelles des paramètres biochimiques des chevaux de traction au Sénégal. L'obtention des valeurs usuelles des paramètres biochimiques aiderait le clinicien à mieux prendre en charge les chevaux de traction sur le plan sanitaire et alimentaire. Et aussi à évaluer l'aptitude et la performance des chevaux de traction par une bonne interprétation des analyses biochimiques. Ces données pourraient être utiles dans le diagnostic des maladies musculaires, rénales et hépatiques.

\section{Remerciements}

Nous remercions, Monsieur le directeur de l'EISMV pour nous avoir facilité les conditions de travail. Nous sommes très reconnaissants aux collègues du laboratoire de Biochimie Dr ROAMBA et Mlle Camille EGUE pour leur aide pour les analyses et les propriétaires de chevaux pour leur collaboration lors de la collecte des échantillons de terrain. Nous exprimons notre gratitude à Dr Justin KOUAMO pour la lecture critique du manuscrit.

\section{REFERENCES}

Akpo Y. 2004. Contribution à l'identification des métiers du cheval dans la région de Dakar et comparaison avec la situation au Maroc. Thèse de Médecine Vétérinaire, Université de Dakar, Sénégal, p. 120.

Bathily A, Sow A, Kalandi M, Mouiche MMM, Sawadogo GJ. 2013. Serum biochemical parameters in racehorses in Senegal. Int. Res. J. Biochem. Bioinform., 3(1): 15-19.

Carlson GP. 2002. Clinical chemistry tests. In Large Animal Internal Medicine, Smith BP (ed). Mosbie: St Louis; 389-414.

Cornus J. 2010. Valeurs usuelles en biochimie sérique chez le cheval selle Français: Données du laboratoire biochimique de l'ENVA. Thèse de Médecine Vétérinaire, Université d'Alfort, France, p. 90.

DIREL (Direction de l'élevage / Ministère de l'Agriculture et de l'Elevage du Sénégal). 2012. Statistique d'élevage : Evolution et répartition des effectifs du cheptel au Sénégal, DIREL.

Evans P. 2010. Body Condition Scoring: A Management Tool for Evaluating All Horses. All Current Publications : 1-5.

Frandson RD, Wilke WL, Fails AD. 2009. Anatomy and Physiology of Farm Animals ( $7^{\text {th }}$ edn). Wiley-Blackwell: Lowa. 
Gálik B, Bíro D, Halo M, Juráček M, Šimko M, Massányi P, Rolinec M. 2012. The effect of different macromineral intakes on mineral metabolism of sport horses. Acta Vet. Brno., 81(2): 113-117.

Guedaoura S, Cabaraux J.F, Moumene A, Tahraoui A, Nicks B. 2011. Evaluation morphométrique de chevaux de race barbe et dérivés en Algérie. Ann. Med. Vet., 155(1): 14-22.

Gul ST, Ahmad M, Khan A, Hussain I. 2007. Haemato-biochemical observations in apparently healthy equine species. Pakistan Vet. J., 27(4): 155-158.

Gurgoze SY, Icen H. 2010. The Influence of Age on Clinical Biochemical Parameters in Pure-bred Arabian Mares. J. Eq. Vet. Sci., 30(10): 569-574.

Ihedioha JI, Agina OA. 2013. Serum biochemistry profile of nigerian horses (equus caballus, linnaeus 1758). Animal Research Intern. J., 10(3): 1826-1833.

Kaneko JJ, Harvey JW, Bruss ML. 2008. Clinical Biochemistry of Domestic Animals ( $6^{\text {th }}$ edn). Academic Press: New York.

Larrat R. 1947. L'élevage du cheval au Sénégal. Revue Élev. Méd. Vét. Pays Trop., 1(4): 256-265.

Niedźwiedź A, Jaworski Z, Filipowski H, Zawadzki M, Wrzosek M, SłużewskaNiedźwiedź M, Nicpoń J. 2013. Serum biochemical reference intervals for the Polish Konik horse (Equus cabalus gmelini Ant.). Vet. Clin. Path., 42(1): 66-69.

Ögren G. 2013. Phosphorus to horses and cows. Licentiate thesis, Swedish University, Uppsala, p. 34.

Petruse C, Falca C. 2008. Sanguine biochemical parameters evolution in Thoroughbred sport horses during training period. Lucrări Stiintifice Medicină Veterinară, XLI(8): 759-763.

Pritchard JC, Burn CC, Barr ARS, Whay HR. 2009. Haematological and serum biochemical reference values for apparently healthy working horses. Pakistan Research in Veterinary Science, 87(3): 389-395.
Rahal K, Guedioura A, Oumouna M. 2009. Paramètres morphométriques du cheval barbe de Chaouchaoua, Algérie. Revue Méd. Vét., 160(12): 586-589.

Rumosa GF, Chimonyo M, Dzama K. 2012. Effect of season and age on blood minerals, liver enzyme levels, and faecal egg counts in Nguni goats of South Africa. Czech J. Anim. Sci., 57(10): 443453.

Simenew K, Gezahegne M, Getachew M, Wondyefraw M, Almayehu L, Eyob I. 2011. References values of clinically important of physiological, hematological, and serum biochemical parameters of apparently healthy working equids of Ethiopia. Global veterinaria., 7(1): 01-07.

Starkey P. 2004. Animal-Powered Transport in Sub-Saharan Africa and Madagascar: Implications for the Changing Roles of Governments and Civil Society. Revue Elev. Méd. Vét. Pays Trop., 57(3-4): 201-208.

Tomenendalova J, Vodicka R, Uhrikova I, Doubek J. 2014. Determination of haematological and biochemical parameters of Przewalski horses (Equus przewalski) kept by the Prague Zoo. Veterinární Medicína., 59(1): 11-21.

Trigo P, Castejon F, Riber C, Muñoz A. 2010. Use of biochemical parameters to predict metabolic elimination in endurance rides. Eq. Vet. J., 42(38): 142-146.

Vall E, Lhoste PH. 2003. Animal power in the West and Central Francophone zone of Africa in a renewed context: the issues for the development achievements. In Working Animals in Agriculture and Transport: A Collection of Some Current research and Development Observations, Pearson RA, Lhoste P, Saastamoinen M, Martin-Rosset W (eds). Wageningen Academic Publisher: Netherlands; 13-25.

Vervuert I, Stanik K, Coenen M. 2006. Effects of different levels of calcium and phosphorus intake on calcium homeostasis in exercising horses. Eq. Vet. J., 36(7): 659-663. 